composed of a combination of hydroxyapatite and tricalcium phosphate have been used in graft procedures on their own.

These materials provide limited mechanical strength and have no inherent bone forming ability. The use of bioceramics is dogged by the problem of variable resorption, which may inhibit rather than promote the ingrowth of bone. ${ }^{11}$ Freshly harvested autologous bone graft remains the material most likely to lead to healing of defects. ${ }^{12}$ The ideal substitute would have the advantage of mechanical strength combined with bone forming capacity through the presence of living osteoblasts and bone morphogenetic proteins. One possible approach would be to use cultured autologous osteoblasts to enhance the osteogenic potential of demineralised bone grafts. ${ }^{13}$

Graft expansion by cell culture has already been described in the treatment of burns. ${ }^{14}$ Autologous epithelial cells were cultured for periods of up to three weeks and then grafted on to areas of skin loss. A 10000 fold increase in available graft material was produced, and $60-80 \%$ of the cultured grafts were successful. The four week delay before autologous keratinocytes become available from culture and the move towards earlier grafting in burns has led to the development of culture of allogeneic fibroblasts and keratinocytes. ${ }^{15}$ In elective orthopaedic and maxillofacial surgery the delay for culture of autologous osteoblasts before grafting would not be the decisive factor. In addition, this delay would allow confirmation of the osteoblast phenotype ${ }^{16}$ which is necessary to exclude contamination by other cell types, usually fibroblasts.

Culturing autologous human osteoblasts on demineralised donor bone may ultimately offer a means of graft enhancement. A small cancellous bone fragment could be harvested percutaneously ${ }^{7}$ under local anaesthesia about four weeks before elective surgery. Osteoblasts obtained in this way could be massively expanded in numbers and cultured on demineralised bone fragments. The result would be a "living" bone graft, which could then be used as needed in definitive surgery, avoiding the problems of harvesting grafts.

PCNOLAN

British Orthopaedic Association Wishbone Research Fellow

R A B MOLLAN

Professor of Orthopaedic Surgery

D J WILSON

Lecturer in Craniofacial Biology

Queen's University of Belfast,

Belfast BT9 7LB

1 Solomon J. Bone grafts. F Bone foint Surg [Br] 1991;73:706-7.

Cockin J. Autologous bone grafting: complications at the donor site. $\mathcal{F}$ Bone foint Surg $[\mathrm{Br}$ 1971:53:153.

Weikel AM, Habal MB. Meralgia paraesthetica: a complication of iliac bone procurement. Plast Reconstr Surg 1977;60:572-4.

4 Glowacki J, Kaban LB, Murray JE, Folkman J, Mulliken JB. Application of the principle of induced osteogenesis for craniofacial defects. Lancet $1981 ; \mathrm{i}: 959-62$.

Sautier JM, Nefussi JR, Boulekbache $H$, Fores $N$. In vitro bone formation on coral granules. In Vitro Cellular and Developmental Biology 1990;26:1079-85.
.

Kirkeby OJ. Revascularisation of bone grafts in rats. $\mathcal{F}$ Bone foint Surg [Br] 1991;73:501-5.

6 Kirkeby OJ. Revascularisation of bone grafts in rats. F Bone foint Surg [Br] 1991;73:501-5. rist M, Jurist J, Dubic F, Strates BS. Quantitation of new bo
implants of bone matrix in rabbits. Clin Orthop 1970;68:247-93.

implants of bone matrix in rabbits. Clin Orthop 1970;68:247-93. Guo MZ, Xia ZS, Lin LB. The mechanical and biological prop

allografts in animals. F Bone foint Surg [Br] 1991;73:791-4.

9 Wang EA, Gerhart TN, Toriumi DM. Bone morphogenetic proteins and developmen Connect Tissue Res 1992;27:154.

10 Burwell R. The function of bone marrow in the incorporation of a bone graft. Clin Orthop $1985 ; 200: 125-41$.

1 Jarcho M. Calcium phosphate ceramics as hard tissue prosthetics. Clin Orthop 1981;157:259-78.

2 Schwarz N, Schlag G, Thurnher M, Eschberger J, Dinges HP, Redi H. Fresh autogenic, frozen allogeneic and decalcified allogeneic bone grafts in dogs. $\mathcal{F}$ Bone foint Surg $[\mathrm{Br}]$ 1991; 73:787-90. Nolan PC, Nicholas RM, Mulholland BJ, Mollan RAB, Wilson DJ. Culture of human osteoblasts on demineralised human bone: a possible means of graft enhancement. $\mathcal{f}$ Bone foint Surg [Br] 1992;74:284-6.

14 Gallico G, O'Connor N, Compton C, Kehinde O, Green H. Permanent coverage of large burn wounds with autologous cultured epithelia. $N$ Engl f Med 1984;16:448.

15 Nanchahal J, Davies D. Cultured composite skin grafts for burns. BMF 1990;301:1342-3.

16 Marie PJ, Lomri A, Sabbagh A, Basle M. Culture and behaviour of osteoblastic cells isolated from normal trabecular bone surfaces. In Vitro Cellular and Development Biology 1989;25:373-80.

17 Salch M. Bone grafting: A percutaneous technique. F Bone foint Surg [Br] 1991;73:867-8.

\title{
Investigating lower bowel symptoms in general practice
}

\section{GPs could do more sigmoidoscopies}

Gastrointestinal problems account for one in 12 consultations in general practice, with lower bowel symptoms accounting for about half of these. ${ }^{1}$ Apart from abdominal emergencies these symptoms include chronic, recurrent abdominal pain; changes in bowel habit; and rectal bleeding. Although self limiting minor conditions account for most of these symptoms, colorectal cancer and inflammatory bowel disease will be responsible for a few. A further large group will, of course, be due to the irritable bowel syndrome.

In this issue Rubin reports that nearly three in four practices in the Northern region possess a proctoscope but only $4 \%$ undertake rigid sigmoidoscopy (p 1542). ${ }^{2}$ Almost half of doctors surveyed thought that proctoscopy and sigmoidoscopy were not appropriate procedures for primary care and that standards might be difficult to maintain. Yet timely investigation of patients with lower bowel problems is important. Rectal bleeding is common, occurring in up to one in six of the general population each year, ${ }^{3}$ and may be the only sign of serious large bowel disease. Colorectal cancer is the second commonest cancer in the United Kingdom, accounting for more than 16000 deaths annually. Survival depends on the stage of disease at diagnosis, and early detection offers the only opportunity to improve survival.
Recognised risk factors for colorectal cancer include a personal history of colorectal neoplasia and inflammatory bowel disease and a family history of colorectal cancer or of one of various inherited disorders that are associated with colorectal cancer. These include familial adenomatous polyposis, site specific cancer of the colon, and the cancer family syndrome (in which colorectal cancer is associated with adenocarcinoma of the breast and genital tract).

To evaluate rectal bleeding anorectal inspection and digital rectal examination are mandatory, although these are not always performed properly. ${ }^{5}$ Patients with rectal bleeding are at low risk of colorectal cancer if they are under 40 and do not have significant personal or family histories. A local anorectal lesion in these patients is a sufficient cause for their bleeding, which may be treated without further investigation. Older patients, those with personal or family histories, and those with recurrent symptoms should be investigated.

Nearly half of all general practitioners have open access to barium enema examinations, and most radiology departments require sigmoidoscopy before contrast radiology. Only a few general practitioners have access to flexible sigmoidoscopy and colonoscopy. ${ }^{7}$ About $70 \%$ of rectal cancers $(15 \%$ of all colorectal malignancies) can be detected by digital rectal 
examination, but $40 \%$ or so of colorectal cancers can be visualised within $25 \mathrm{~cm}$ on rigid sigmoidoscopy and $60 \%$ within $60 \mathrm{~cm}$ on flexible sigmoidoscopy. ${ }^{8-10}$

Should general practitioners be encouraged to acquire the equipment and skill to perform sigmoidoscopy or would resources be better used in providing fast track access to rigid or flexible sigmoidoscopy or colonoscopy in an outpatient setting? ${ }^{11-13}$ Although the latter would seem to offer an ideal service for patients and result in earlier and more accurate evaluation of lower bowel symptoms, advocates of practice based investigation assert that rigid, and possibly flexible, sigmoidoscopy is within the scope of most general practices. ${ }^{1+15}$ The equipment required for rigid sigmoidoscopy is not expensive, although flexible sigmoidoscopes are costly; neither investigation attracts payment as a minor surgical procedure. Performing these investigations in general practice would benefit patients, avoiding the delay and anxiety associated with an outpatient visit, and may use resources more effectively, particularly in fundholding practices. ${ }^{16}$

Concerns must, however, exist about training and skill and the accuracy of investigations performed relatively infrequently - even if one partner undertakes the procedure in a large group practice. Patients with suspicious rectal bleeding in whom rigid sigmoidoscopy is non-contributory will still require referral to hospital, as will those in whom colorectal polyps or cancer are identified. Initially patients with inflammatory bowel disease may be managed in general practice, but some will eventually require specialist supervision.

Many general practitioners have special skills in managing disease. Restricting their activity would not only be demoralising but would run counter to the trend towards long term management of important medical conditions in general practice. Few general practitioners want to become mere signposts to hospital, and many regard the provision of increasingly comprehensive medical care as a priority in practice development.

General practitioners should be able to decide whether they want to provide these kinds of services for their patients. This has clear implications for adequate instruction during vocational training and for recognising sigmoidoscopy as a minor surgical procedure. Within a health district it would be reasonable for some general practitioners to develop sigmoidoscopy in their practice; others would be supported by outpatient services sensitive to the need for speedy investigation of patients at high risk of colorectal malignancy. In these services the provision of sigmoidoscopy and colonoscopy would be determined by discussion between general practitioners and specialists.

William Leech Professor of Primary Health Care,

ROGER JONES

Primary Health Care,

The Medical School,

Newcastle upon Tyne NE2 4HH

1 Royal College of General Practitioners, Office of Population Censuses and Surveys, Department of Health and Social Security. Morbidity statistics from general practice 1981-82. London: HMSO, 1990. (Series MB5 No 2 .)

2 Rubin GP. Endoscopy facilities in general practice. BMf 1992;304:1542-3.

3 Jones R, Lydeard S. Irritable bowel syndrome in the general population. $B M \mathcal{f}$ 1992;304:87-90.

4 Murday V, Slack J. Inherited disorders associated with colorectal cancer. Cancer Surv 1989;8: $139-57$.

Springall RG, Todd IP. General practitioner referral of patients with lower gastrointestinal symptoms. I R Soc Med 1988:81:87-8.

6 Dixon AR, Thornton-Holmes J, Cheetham N. General practitioners' awareness of colorectal cancer: a 10 year review. BMF 1990;301:152-3.

7 Bramble MG. Open access endoscopy - a nationwide survey of current practice. Gut 1992;33. $282-5$

8 Moore MR. Cancer of the colon. In: Hurst JW, ed. Medicine for the practising physician. Boston Butterworths, 1983:76-80.

9 Winawer SJ, Cummins R, Baldwin MP, Ptak A. A new flexible sigmoidoscope for the generalis Gastrointest Endosc 1982;28:233-6

10 Berg JW, Howell MA. The geographic pathology of bowel cancer. Cancer 1974;34:807-14

11 Kalra L, Price WR, Jones BJM, Hamlyn AN. Open access fibresigmoidoscopy: a comparative audit of efficacy. BM7 1988;296:1095-6.

2 Vellacot KD, Roe AM, Mortensen NJMcC. An evaluation of a direct access flexible fibreoptic sigmoidoscopy service. Ann R Coll Surg Engl 1987;69:149-52.

3 Tate JJT, Royle GT. Open access colonoscopy for suspected colonic neoplasm. Gut 1988;29: $1322-5$.

14 Hocut JE, Hainer BL, Jackson MG. Flexible fibreoptic sigmoidoscopy; its use in family medicine. 7 Am Board Fam Pract 1988;1:189-93.

15 Rodney WM. Procedural skills in flexible sigmoidoscopy and colonoscopy for the family physician. Primary Care 1988;15:79-91.

16 Cox T. Flexible sigmoidoscopy in general practice. In: Pereira Gray DJ, ed. Royal College of General Practitioners members handbook. London: Royal College of General Practitioners, 1991:345-7.

\section{Cardiac imaging with radionuclides}

\section{A useful addition to electrocardiography and echocardiography}

Advances in cardiovascular imaging have made accurate diagnosis of cardiac disease easier. Cardiologists, particularly those in teaching centres, now have a wide range of radionuclide techniques to supplement clinical examination, electrocardiography at rest and during exercise, and echocardiography. These include radionuclide ventriculography, perfusion imaging, infarct avid imaging, and, in the most privileged locations, positron emission tomography.

Although echocardiography is already available in most district general hospitals, cardiac radionuclide investigation is not. Only half of the health districts in England and Wales perform cardiac radionuclide investigation'; a recent survey found that the rate of such investigation in Britain was one fifth that in the United States. ${ }^{2}$ This reflects the limited facilities for this technique but also the lack of importance that British physicians place on these tests.

Determining the optimal use of these techniques is difficult, and American studies suggest a high rate of inappropriate use. ${ }^{34}$ Good comparative studies are still needed to clarify each technique's role in diagnosis, but in their absence doctors should at least be aware of cardiac radionuclide investigations and understand their principles, main uses, and limitations.

Ventriculography, with a radionuclide that is retained within the blood pool, can be used to estimate ejection fraction, one of the most important determinants of prognosis, both in chronic ischaemic heart disease and after myocardial infarction. ${ }^{56}$ Unlike echocardiography, a radionuclide study is not dependent on finding an adequate ultrasonic window. Although anatomical detail is much better with ultrasonography, the isotope technique has the advantage of readily assessing left ventricular function, even in the presence of akinetic or dyskinetic segments. Atrial fibrillation, however, reduces the reliability of both techniques, particularly if the ventricular rate is not well controlled.

Myocardial perfusion imaging depends on the use of agents such as thallium-201, which are taken up by perfused myocardium in proportion to blood flow. Ischaemic or infarcted muscle appears as a "cold spot." In this test perfusion images obtained immediately after stress (usually exercise) are compared with those at rest. The quality of the image is very important for interpreting the results and has 